\title{
Simple finite field method for calculation of static and dynamic vibrational hyperpolarizabilities: Curvature contributions
}

\author{
Bernard Kirtman and Josep M. Luis ${ }^{\text {a) }}$ \\ Department of Chemistry, University of California, Santa Barbara, California 93106 \\ David M. Bishop \\ Department of Chemistry, University of Ottawa, Ottawa K1N 6N5, Canada
}

(Received 29 December 1997; accepted 20 March 1998)

\begin{abstract}
In the static field limit, the vibrational hyperpolarizability consists of two contributions due to: (1) the shift in the equilibrium geometry (known as nuclear relaxation), and (2) the change in the shape of the potential energy surface (known as curvature). Simple finite field methods have previously been developed for evaluating these static field contributions and also for determining the effect of nuclear relaxation on dynamic vibrational hyperpolarizabilities in the infinite frequency approximation. In this paper the finite field approach is extended to include, within the infinite frequency approximation, the effect of curvature on the major dynamic nonlinear optical processes. (C) 1998 American Institute of Physics. [S0021-9606(98)02224-7]
\end{abstract}

\section{INTRODUCTION}

The design of materials with large nonlinear optical (NLO) properties is currently of great interest, ${ }^{1-3}$ mainly because of potential utilization in a variety of optical and electro-optical devices. At the molecular level these NLO properties are determined by the dynamic hyperpolarizabilities. Although one might, at first, think that dynamic hyperpolarizabilities are primarily electronic in origin, there is a growing body of evidence that, for materials with large NLO properties, the vibrations play an important role. Indeed, a number of cases exist ${ }^{4-7}$ where the vibrational hyperpolarizability far exceeds the electronic hyperpolarizability.

A perturbation treatment of dynamic vibrational hyperpolarizabilities has been given by Bishop and Kirtman ${ }^{8,9}$ (BK). This treatment is based on the general sum-over-states formulas ${ }^{10}$ for the total hyperpolarizability given in terms of vibronic energies and dipole moment matrix elements. The vibrational and electronic contributions are, then, separated by applying a canonical, or clamped nucleus, approximation ${ }^{11}$ wherein the electronic and vibrational motions are considered sequentially rather than simultaneously. BK express the resulting vibrational terms using a double perturbation expansion in orders $(n, m)$ of electrical and mechanical anharmonicity, respectively. In low order, i.e., $(n, m)$ $=(0,0),(1,0),(0,1),(1,1)$, and $(2,0)$, this leads to a set of compact expressions ${ }^{9}$ for the dynamic vibrational hyperpolarizabilities.

Quite often the vibrational hyperpolarizability is estimated in the double harmonic approximation, ${ }^{4,5,12,13}$ i.e., $(n, m)=(0,0)$, but there are also a number of studies ${ }^{14-29}$ where anharmonic contributions have been evaluated. Although these latter studies mostly pertain to the static hyperpolarizability, they do demonstrate that going beyond the

\footnotetext{
a) Permanent address: Institute of Computational Chemistry and Department of Chemistry, University of Girona, 17017 Girona, Catalonia, Spain.
}

double harmonic approximation can be important and that the convergence behavior will vary dramatically from one system to another.

There have been two impediments to including anharmonicities in dynamic vibrational hyperpolarizability calculations. First, the BK compact formulas were, until now, ${ }^{30}$ complete only through first order (i.e., $n+m=1$ ). Probably of more importance, particularly for NLO materials which usually involve large molecules or polymers, the anharmonicity constants that are required are often computationally burdensome to evaluate.

It is possible to circumvent these difficulties in two special cases through the use of finite field (FF) methods whereby various molecular properties are determined as a function of one or more static applied electric fields. One special case is the vibrational hyperpolarizability in the static limit, ${ }^{22,24}$ which satisfies the relation

$$
\begin{aligned}
& \text { vibrational hyperpolarizability +ZPVA } \\
& \text { = nuclear relaxation contribution } \\
& + \text { curvature contribution. }
\end{aligned}
$$

Here the vibrational hyperpolarizability is the quantity discussed in BK, and ZPVA refers to the zero-point vibrational averaging correction for the electronic hyperpolarizability (which depends upon the nuclear coordinates). On the righthand side (rhs) of Eq. (1), the nuclear relaxation contribution $^{24}$ arises from the change in the electronic energy, or dipole moment, due to the field-induced relaxation of the molecular geometry. The origin of the curvature contribution ${ }^{24}$ is the change in zero-point vibrational energy caused directly by the field and indirectly by the geometry relaxation. For a diatomic molecule it has already been demonstrated $^{31}$ that Eq. (1) is valid. The ZPVA term, in particular, is part of the curvature contribution. An extension of the proof for diatomics to an arbitrary polyatomic 
molecule,$^{30}$ building on the treatment given recently by Luis et al. ${ }^{16}$ is presented in the next paper. The relationship between the alternative approaches implied by the two sides of Eq. (1) has recently been reviewed. ${ }^{32}$

The other special case is the infinite frequency limit. In that limit the nuclear relaxation contribution is obtained by considering the effect of field-induced geometry relaxation on the linear polarizability $(\alpha)$ and the first hyperpolarizability $(\beta)$, rather than on the electronic energy or dipole moment. As Bishop, Hasan, and Kirtman (BHK) have shown, ${ }^{33}$ this yields the leading perturbation terms of each type ${ }^{34}$ in the formulas for the most common NLO processes. In the few examples that have been examined, ${ }^{35}$ it has been found that the vibrational NLO property at a typical optical frequency is not very different from the value at the infinite frequency limit. Thus, the latter constitutes a useful approximation.

The primary purpose of the current paper is to extend the FF/infinite frequency method to include the analog of the curvature contribution in Eq. (1). We find that instead of the electronic $\alpha$ and $\beta$, which yield the nuclear relaxation contribution, one must now use the ZPVA correction to these properties. Our treatment then follows along exactly the same lines as BHK and gives entirely analogous results, as will be seen from the analysis carried out in the next section.

The leading terms in the ZPVA correction for $\alpha$ and $\beta$ are first order in electrical or mechanical anharmonicity. However, the required anharmonicity constants have repeated indices and, therefore, can be determined with only slightly more computational effort than is necessary for the harmonic parameters. This is discussed in Sec. III, along with other approximate computational simplifications. In addition, the finite field approach has certain limitations from a theoretical and interpretive point of view. Methods to reduce these limitations are also considered in Sec. III and, finally, we close with a summary of our results.

\section{ANALYSIS}

We let the equilibrium geometry in an applied electric field, $\mathbf{F}$, be denoted by $\mathbf{R}_{F}$, while $P^{e}\left(\mathbf{F}^{\prime}, \mathbf{R}_{F}\right)$ is the value of the electronic property $P^{e}$ calculated at $\mathbf{R}_{F}$ in the presence of a field $\mathbf{F}^{\prime}$. In the following, $\mathbf{F}$ and $\mathbf{F}^{\prime}$ will always be the same, although this is not required. The field-dependent vibrationally averaged value of $P^{e}$ is given by

$$
\left\langle 0_{F}\left|P^{e}(\mathbf{F}, \mathbf{R})\right| 0_{F}\right\rangle=P^{e}\left(\mathbf{F}, \mathbf{R}_{F}\right)+\Delta P^{\mathrm{ZPVA}}\left(\mathbf{F}, \mathbf{R}_{F}\right),
$$

where

$$
\Delta P^{\mathrm{ZPVA}}\left(\mathbf{F}, \mathbf{R}_{F}\right)=\left\langle 0_{F}\left|P^{e}(\mathbf{F}, \mathbf{R})-P^{e}\left(\mathbf{F}, \mathbf{R}_{F}\right)\right| 0_{F}\right\rangle .
$$

Here $\mathbf{R}$ is an arbitrary geometry and $\left|0_{F}\right\rangle$ is the fielddependent ground-state vibrational wave function. The BHK $\mathrm{FF} /$ nuclear relaxation method is based on the first term on the rhs of Eq. (2). One defines the difference

$$
\left(\Delta P^{e}\right)_{\mathbf{R}_{F}}=P^{e}\left(\mathbf{F}, \mathbf{R}_{F}\right)-P^{e}\left(\mathbf{0}, \mathbf{R}_{0}\right)
$$

and expands this quantity as a Taylor series in $\mathbf{F}$. For $P^{e}$ $=\mu^{e}, \alpha^{e}, \beta^{e}$, this leads to

$$
\begin{aligned}
& \left(\Delta \mu_{\alpha}^{e}\right)_{R_{F}}=a_{1}^{e} F_{\beta}+\frac{1}{2} b_{1}^{e} F_{\beta} F_{\gamma}+\frac{1}{6} g_{1}^{e} F_{\beta} F_{\gamma} F_{\delta}+\ldots, \\
& \left(\Delta \alpha_{\alpha \beta}^{e}\right)_{\mathbf{R}_{F}}=b_{2}^{e} F_{\gamma}+\frac{1}{2} g_{2}^{e} F_{\gamma} F_{\delta}+\ldots \\
& \left(\Delta \beta_{\alpha \beta \gamma}^{e}\right)_{\mathbf{R}_{F}}=g_{3}^{e} F_{\delta}+\ldots
\end{aligned}
$$

with

$$
\begin{aligned}
& a_{1}^{e}=\alpha_{\alpha \beta}^{e}(0 ; 0)+\alpha_{\alpha \beta}^{n r}(0 ; 0), \\
& b_{1}^{e}=\beta_{\alpha \beta \gamma}^{e}(0 ; 0,0)+\beta_{\alpha \beta \gamma}^{n r}(0 ; 0,0), \\
& g_{1}^{e}=\gamma_{\alpha \beta \gamma \delta}^{e}(0 ; 0,0,0)+\gamma_{\alpha \beta \gamma \delta}^{n r}(0 ; 0,0,0), \\
& b_{2}^{e}=\beta_{\alpha \beta \gamma}^{e}(0 ; 0,0)+\beta_{\alpha \beta \gamma}^{n r}(-\omega ; \omega, 0)_{\omega \rightarrow \infty}, \\
& g_{2}^{e}=\gamma_{\alpha \beta \gamma \delta}^{e}(0 ; 0,0,0)+\gamma_{\alpha \beta \gamma \delta}^{n r}(-\omega ; \omega, 0,0)_{\omega \rightarrow \infty}, \\
& g_{3}^{e}=\gamma_{\alpha \beta \gamma \delta}^{e}(0 ; 0,0,0)+\gamma_{\alpha \beta \gamma \delta}^{n r}(-2 \omega ; \omega, \omega, 0)_{\omega \rightarrow \infty} .
\end{aligned}
$$

In Eqs. (8)-(10) we have used the standard notation, e.g., $\gamma\left(-\omega_{\sigma} ; \omega_{1}, \omega_{2}, \omega_{3}\right)$, to designate the frequencies of the oscillating electric fields (in the order $F_{\alpha}, F_{\beta}, F_{\gamma} \cdots$ ) and, as usual, $\omega_{\sigma}=\Sigma_{i} \omega_{i}$. The value obtained for each quantity is that at the field-free equilibrium geometry $\mathbf{R}_{0}$.

Although all the calculations are done with static fields, Eqs. (9) and (10) yield dynamic NLO properties in the nuclear relaxation infinite frequency $(n r / \omega \rightarrow \infty)$ approximation. Thus, $\beta_{\alpha \beta \gamma}^{n r}(-\omega ; \omega, 0)_{\omega \rightarrow \infty}$ contributes to the Pockels effect, $\gamma_{\alpha \beta \gamma \delta}^{n r}(-\omega ; \omega, 0,0)_{\omega \rightarrow \infty}$ to the Kerr effect, and $\gamma_{\alpha \beta \gamma \delta}^{n r}(-2 \omega ; \omega, \omega, 0)_{\omega \rightarrow \infty}$ to dc-second harmonic generation (dc-SHG). Analytical expressions for the terms included in the $n r / \omega \rightarrow \infty$ approximation can be obtained ${ }^{16}$ from a double expansion about $\left(0, \mathbf{R}_{0}\right)$ of the electronic energy, $V(\mathbf{F}, \mathbf{Q})$, in terms of the field vector $\mathbf{F}=\left(F_{x}, F_{y}, F_{z}\right)$ and the normal coordinate displacements, $\mathbf{Q}$. For a fixed $\mathbf{F}$ this double expansion yields $\mathbf{R}_{F}$ and, then, subsequent variations of $F_{x}, F_{y}, F_{z}$ give the coefficients in Eqs. (5)-(7). Exactly the same expressions can be derived from the BK perturbation treatment by taking the lowest order terms of each type that survive after letting $\omega$ become infinite. These terms are listed in Table I of BHK and the connections with the double expansion method are given in Table I of Ref. 16. The two approaches together yield a definitive interpretation of the quantities in Eqs. (9) and (10).

From the BK perturbation treatment it is easy to demonstrate that the nuclear relaxation (and curvature) contribution to vibrational second and third harmonic generation (SHG and THG), i.e., $\quad \beta_{\alpha \beta \gamma}^{v}(-2 \omega ; \omega, \omega)_{\omega \rightarrow \infty}$ and $\gamma_{\alpha \beta \gamma \delta}^{v}$ $(-3 \omega ; \omega, \omega, \omega)_{\omega \rightarrow \infty}$, vanishes in the infinite frequency limit. Finally, in the $n r / \omega \rightarrow \infty$ approximation the vibrational intensity-dependent refractive index (IDRI), i.e., $\gamma_{\alpha \beta \gamma \delta}^{n r}$ $(-\omega ; \omega,-\omega, \omega)_{\omega \rightarrow \infty}$ contains just the $\left[\alpha^{2}\right]^{(0,0)}$ perturbation term (see Tables I and II of Ref. 30), which may be estimated through first order in the finite field method [see Eq. (12) of BHK] by combining $\gamma_{\alpha \beta \gamma \delta}^{n r}(-\omega ; \omega, 0,0)_{\omega \rightarrow \infty}, \quad \gamma_{\alpha \beta \gamma \delta}^{n r}$ $(-2 \omega ; \omega, \omega, 0)_{\omega \rightarrow \infty}$, and $\gamma_{\alpha \beta \gamma \delta}^{n r}(0 ; 0,0,0)_{\omega \rightarrow \infty}$. In this way we account for the major NLO processes. However, the IDRI estimate is valid only for the diagonal tensor components or for the mean value.

Next we consider the second, or ZPVA, term in Eq. (2). In this case application of the finite field method as above 
yields the vibrational curvature $/ \omega \rightarrow \infty$ contribution to the various NLO processes. Unlike nuclear relaxation, the curvature contribution is not limited to low orders of perturbation theory. It is convenient, however, to begin with the lowest order terms in Eq. (3) which are given by

$$
\Delta P^{\mathrm{ZPVA}}=[P]^{0,1}+[P]^{1,0},
$$

with

$$
[P]^{0,1}=-\frac{\hbar}{4} \sum_{a}\left(\sum_{b} \frac{F_{a b b}}{\omega_{b}}\right)\left(\frac{\partial P / \partial Q_{a}}{\omega_{a}^{2}}\right)
$$

and

$$
[P]^{1,0}=\frac{\hbar}{4} \sum_{a} \frac{\left(\partial^{2} P / \partial Q_{a}^{2}\right)}{\omega_{a}} .
$$

Now we take the difference

$$
\Delta P^{\mathrm{ZPVA}}\left(\mathbf{F}, \mathbf{R}_{F}\right)-\Delta P^{\mathrm{ZPVA}}\left(\mathbf{0}, \mathbf{R}_{0}\right),
$$

where $\Delta P^{\mathrm{ZPVA}}\left(\mathbf{0}, \mathbf{R}_{0}\right)$ is defined by Eq. (3) with $\mathbf{F}=0$, and expand that quantity as a power series in the field(s). This leads to a set of relations analogous to Eqs. (5)-(7) except that one must use $\Delta P^{\mathrm{ZPVA}}\left(\mathbf{F}, \mathbf{R}_{F}\right)-\Delta P^{\mathrm{ZPVA}}\left(\mathbf{0}, \mathbf{R}_{0}\right)$ instead of $\left(\Delta P^{e}\right)_{\mathbf{R}_{F}}$. Similarly, in Eqs. (8)-(10) we substitute $\Delta P^{\mathrm{ZPVA}}$ for $P^{e}$ and simultaneously replace " $n r$ " by "curv." In replacing " $n r$ " with "curv" it should be understood that the resulting quantity is the difference between the total curvature contribution and that due to $\Delta P^{\mathrm{ZPVA}}$. Of course, for $\alpha, \beta$, etc., $\Delta P^{\mathrm{ZPVA}}$ vanishes at the infinite frequency limit.

In order to verify that the ZPVA/curvature relations are correct we have, again, combined the double expansion and BK perturbation methods. In fact, using Eq. (11) it is easy to show (see the Appendix) that

$$
\begin{aligned}
\beta_{\alpha \beta \gamma}^{\text {curv }}(-\omega ; \omega, 0)_{\omega \rightarrow \infty}= & {[\mu \alpha]_{\omega \rightarrow \infty}^{\mathrm{II}}, } \\
\gamma_{\alpha \beta \gamma \delta}^{\mathrm{curv}}(-\omega ; \omega, 0,0)_{\omega \rightarrow \infty}= & {\left[\alpha^{2}\right]_{\omega \rightarrow \infty}^{\mathrm{II}}+[\mu \beta]_{\omega \rightarrow \infty}^{\mathrm{II}} } \\
& +\left[\mu^{2} \alpha\right]_{\omega \rightarrow \infty}^{\mathrm{III}}, \\
\gamma_{\alpha \beta \gamma \delta}^{\mathrm{curv}}(-2 \omega ; \omega, \omega, 0)_{\omega \rightarrow \infty} & =[\mu \beta]_{\omega \rightarrow \infty}^{\mathrm{II}},
\end{aligned}
$$

where we have used the shorthand []$^{\mathrm{II}}=[]^{2,0}+[]^{1,1}$ +[]$^{0,2}$ and []$^{\mathrm{III}}=[]^{3,0}+[]^{2,1}+[]^{1,2}+[]^{0,3}$. Note that the square bracket quantities depend explicitly on the NLO process, although this has not been indicated. Thus, for example, $[\mu \beta]_{\omega \rightarrow \infty}^{\mathrm{II}}$ in Eq. (16) is not the same as $[\mu \beta]_{\omega \rightarrow \infty}^{\mathrm{II}}$ in Eq. (17).

Until recently, ${ }^{30}$ the compact BK perturbation formulas of order $(0,2)$ have not been available and formulas for total order $n+m \geqslant 3$ are still not known. One can, nonetheless, verify Eqs. (15)-(17) with recourse to known formulas. If the static perturbation expression is available (as it always is), then the infinite frequency formula is readily obtained without knowing the general frequency-dependent result. As far as the diagonal tensor components (and the mean values) are concerned, the static and infinite frequency expressions are identical except for multiplicative constants, which depend only on the NLO process and the type of perturbation term. This means, for instance, that the $\left[\mu^{2} \alpha\right]_{\omega \rightarrow \infty}^{\mathrm{III}}$ term in Eq. (16) can be found from the corresponding $\left[\mu^{2} \alpha\right]_{\omega=0}^{\mathrm{III}}$, $\left[\mu^{2} \alpha\right]_{\omega \rightarrow \infty}^{\mathrm{I}}$ and $\left[\mu^{2} \alpha\right]_{\omega=0}^{\mathrm{I}}$ terms.

The next lowest order terms in $\Delta P^{\mathrm{ZPVA}}$ are two orders higher than those given in Eq. (11), i.e.,

$$
\Delta P^{\mathrm{ZPVA}}=[P]^{\mathrm{I}}+[P]^{\mathrm{III}} .
$$

Thus, the infinite frequency curvature results obtained by the finite field method are correct through the second (total) order of perturbation theory. It is unlikely that Eq. (18) will be utilized in the foreseeable future with standard quantum chemistry programs since the evaluation of $[P]^{0,3}$ requires quintic force constants and $[P]^{3,0}$ requires the fourth derivative of the electronic property. Without doing any further analysis we know that the additional terms in Eqs. (15)-(17) would be of the same type but two orders higher than those already discussed.

On the other hand, it may be feasible to evaluate the total ZPVA correction directly by techniques that sample the complete potential energy surface. Taking that idea one step further, one could determine the total electronic property as given by Eq. (2). Then the entire vibrational hyperpolarizability, including both the nuclear relaxation and curvature contributions, would be obtained at the same time rather than stepwise, as in the current procedure.

\section{DISCUSSION}

The starting point for a FF/curvature calculation is the ZPVA correction term given, in lowest order, by Eqs. (11)(13). It is important to note that only a subset of the mechanical and electrical anharmonicity constants, i.e., those with repeated indices, are needed for evaluation of $[P]^{0,1}$ and $[P]^{1,0}$. Once the equilibrium geometry and normal coordinates have been determined, then the required anharmonic force constants can be obtained by taking the diagonal second derivatives of the energy gradients $\partial V / \partial Q_{a}=-F_{a}$,

$$
F_{a b b}=-\lim _{Q_{b} \rightarrow 0} \frac{\left[F_{a}\left(+Q_{b}\right)+F_{a}\left(-Q_{b}\right)\right]}{Q_{b}^{2}} .
$$

From the numerical point of view, it is clear that the computational effort that must be expended to obtain the set of cubic force constants, $F_{a b b}$, is similar to that involved in calculating the set of quadratic force constants, $F_{a b}$. A similar conclusion applies to analytical differentiation assuming 
that an appropriate computer code is available. Essentially the same analysis also pertains to the anharmonic electronic property derivatives $\partial^{2} P / \partial Q_{a}^{2}$.

Beyond the simplifications discussed above we can also consider various approximations that may reduce the computational effort. One possibility is to carry out preliminary calculations at a lower level than desired in terms of electronic structure method and/or basis set. These preliminary calculations could be used to specify the normal modes and, perhaps, to eliminate some of these modes as being relatively unimportant. An investigation ${ }^{36}$ of this approach for the case of nuclear relaxation has given promising initial results.

The finite field approach to dynamic vibrational hyperpolarizabilities has certain limitations that should be borne in mind. First of all, it is only valid in the infinite frequency limit. In that limit the electronic properties and their zeropoint vibrational averages vanish. In order to estimate the (zero-field) $\Delta P^{\mathrm{ZPVA}}$ at an optical frequency, we can scale the static value in the same manner often used in treating electron correlation. ${ }^{37}$ An example is afforded by a recent treatment ${ }^{38}$ of the mean dc-second harmonic generation in methane. The error due to the difference between the frequency-dependent and the static $\Delta P^{\mathrm{ZPVA}}$ was calculated to be $3.5 \%$ of the corresponding electronic property at $\omega$ $=0.06$ a.u. This error grows to $10.1 \%$ at $\omega=0.10 \mathrm{au}$, but is reduced by a factor of 2 when the static $\Delta P^{\mathrm{ZPVA}}$ is scaled. From the few other examples available $\mathrm{e}^{39-42}$ it appears that, if anything, methane corresponds to a worst case scenario.

The error in the infinite frequency approximation for nuclear relaxation has been examined by Bishop and Dalskov. ${ }^{35}$ For five small molecules they evaluated the mean dc-SHG, THG, and IDRI, as well as the isotropic and anisotropic Kerr effect at the $\mathrm{He} / \mathrm{Ne}$ laser frequency $(\omega$ $=0.072$ a.u.) and compared them with the same properties at $\omega \rightarrow \infty$. In those cases where nuclear relaxation is important, the maximum error $\left(\mathrm{NH}_{3}\right.$; anisotropic Kerr effect) due to the infinite frequency approximation (i.e., their approximation $B$ ) turns out to be less than $12 \%$ of the $\omega=0.072$ value.

Another aspect of the finite field approach, which is a limitation on the one hand and an advantage on the other, is the fact that one obtains the entire curvature (or nuclear relaxation) effect without knowing the contribution of individual terms (cf. Ref. 16). For purposes of analysis, however, there are (at least) three different ways of dividing up the total contribution that could prove useful: (1) $[P]^{0,1}+[P]^{1,0} ;(2)$ individual normal coordinates $Q_{a}$ in Eqs. (12) and (13); and (3) individual normal (or internal) coordinates in the field-dependent geometry optimization. All three of these divisions can be carried out separately or in concert.

In implementing our FF/curvature procedure it should be borne in mind that all the quantities in Eqs. (12), (13), and (19) are field dependent. That includes the normal coordinates, $Q_{a}$, and the vibrational frequencies, $\omega_{a}$, as well as the forces, $F_{a}$, and the electronic properties, $P$. For a given field one can use standard quantum chemistry programs to determine the field-dependent equilibrium geometry and all $Q_{a}$, $\omega_{a}$ and $P=\alpha$ or $\beta$ (note that $F_{a}=0$ ). Then, with the field fixed, the required derivatives of $P$ and $F_{a}$ can be calculated using finite displacements of the $Q_{a}$. Finally, $b_{2}^{\mathrm{ZPVA}}, g_{2}^{\mathrm{ZPVA}}$, and $g_{3}^{\mathrm{ZPVA}}$ are found by varying the field and fitting the quantity in Eq. (14) to the analog of either Eq. (6) or Eq. (7).

In summary, the combination of this paper with BHK yields a simple, practical FF method for calculating vibrational NLO properties in the infinite frequency approximation. At the lowest level of treatment the results are complete through second-order perturbation theory (and include some of the higher-order terms), where we refer here to the total order in mechanical and electrical anharmonicity. An analysis of existing data suggests that the infinite frequency approximation will be adequate in most instances.

\section{ACKNOWLEDGMENTS}

D.M.B. acknowledges financial support from the Natural Sciences and Engineering Research Council of Canada. J.M.L. was supported by the Generalitat de Catalunya through the CIRIT Project No. FI/95-5101.

\section{APPENDIX}

In this Appendix we outline the derivation of Eq. (15); Eqs. (16) and (17) may be obtained in an analogous fashion. Our starting point is the lowest order expression for the static $\Delta \alpha^{\mathrm{ZPVA}}=\Delta \alpha^{\mathrm{ZPVA}}(0 ; 0)$ as given by Eqs. (11)-(13). In the notation of Ref. 17,

$$
\begin{aligned}
\Delta \alpha_{\alpha \beta}^{\mathrm{ZPVA}}(0 ; 0)= & -\sum_{i=1}^{3 N-6}\left(2 a_{20}^{i i}\right)^{-1 / 2} \\
& \times\left[a_{22}^{i i, \alpha \beta}-3 \sum_{j=1}^{3 N-6} a_{30}^{i i j} q_{2}^{j, \alpha \beta}\right],
\end{aligned}
$$

where

$$
\begin{aligned}
& a_{n m}^{i_{1} \cdots i_{n}, j_{1} \cdots j_{m}} \\
& \quad=\frac{1}{n ! m !}\left(\frac{\partial^{(n+m)} V\left(Q_{1}, \ldots, Q_{3 N-6}, F_{x}, F_{y}, F_{z}\right)}{\partial Q_{i_{1}} \cdots \partial Q_{i_{n}} \partial F_{j_{1}} \cdots \partial F_{j_{m}}}\right)_{Q, F} \\
& \quad \text { and } q_{2}^{\lambda, \alpha \beta}=a_{12}^{\lambda, \alpha \beta} / 2 a_{20}^{\lambda \lambda} .
\end{aligned}
$$

Instead of evaluating the potential energy derivatives in Eq. (A2) at $\mathbf{Q}=0, \mathbf{F}=0$ as done previously, we regard them as a function of the electric field, $\mathbf{F}$, and the field-free normal coordinates, $\mathbf{Q}$. In order to carry out an expansion in $\mathbf{F}$ at the field-dependent equilibrium geometry $\mathbf{R}_{F}$, as desired, we follow the same two-step iterative procedure described in Refs. 16 and 17: (1) the stationary condition $(\partial V / \partial Q)_{\mathbf{R}_{F}}$ $=0$ is applied to find $\mathbf{R}_{F}$, and, then, (2) $V$ is determined as a function of $\mathbf{F}$ at that geometry. In doing this it is important to correctly take into account the dependence of the vibrational force constants on $\mathbf{F}$ as discussed in the following article. Indeed, the same field-dependent unitary transformation that diagonalizes the harmonic force constant matrix must be applied to all the other $a_{n m}$ coefficients. Then, straightforward algebra leads to the first derivative, 


$$
\begin{aligned}
& \frac{\partial\left[\Delta \alpha_{\alpha \beta}^{\mathrm{ZPVA}}(0 ; 0)\right]}{\partial F_{\gamma}}=-\frac{1}{2} \sum_{i=1}^{3 N-6}\left(2 a_{20}^{i i}\right)^{1 / 2}\left[6 a_{23}^{i i, \alpha \beta \gamma}-18 \sum_{j=1}^{3 N-6} \alpha_{30}^{i i j} q_{3}^{j, \alpha \beta \gamma}-6 \sum_{j=1}^{3 N-6} a_{32}^{i i j, \alpha \beta} q_{1}^{j, \gamma}-6 \sum_{j=1}^{3 N-6} a_{31}^{i i j, \gamma} q_{2}^{j, \alpha \beta}\right. \\
& +24 \sum_{j, k=1}^{3 N-6} a_{40}^{i i j k} q_{1}^{j, \gamma} q_{2}^{k, \alpha \beta}+6 \sum_{j, k=1}^{3 N-6} a_{30}^{i i j} a_{22}^{j k, \alpha \beta} q_{1}^{k, \gamma} / a_{20}^{j j}+6 \sum_{j, k=1}^{3 N-6} a_{30}^{i i j} a_{21}^{j k, \gamma} q_{2}^{k, \alpha \beta} / a_{20}^{j j} \\
& -18 \sum_{j, k, l=1}^{3 N-6} a_{30}^{i i j} a_{30}^{j k l} q_{2}^{k, \alpha \beta} q_{1}^{l, \gamma}-4 \sum_{j=1}^{3 N-6} a_{22}^{i j, \alpha \beta} a_{21}^{i j, \gamma} / A_{20}^{i j}+24 \sum_{j, k=1}^{3 N-6} a_{22}^{i j, \alpha \beta} a_{30}^{i j k} q_{1}^{k, \gamma} / A_{20}^{i j} \\
& \left.+24 \sum_{j, k=1}^{3 N-6} a_{21}^{i j, \gamma} a_{30}^{i j k} q_{2}^{k, \alpha \beta} / A_{20}^{i j}-72 \sum_{j, k, l=1}^{3 N-6} a_{30}^{i j k} a_{30}^{i j l} q_{2}^{k, \alpha \beta} q_{1}^{l, \gamma} / A_{20}^{i j}\right]
\end{aligned}
$$

where

$$
\begin{aligned}
& A_{20}^{i j}=2\left(\sqrt{a_{20}^{i i}}+\sqrt{a_{20}^{j j}}\right)^{2}, \\
& q_{1}^{\lambda, \alpha}=a_{11}^{\lambda, \alpha} / 2 a_{20}^{\lambda \lambda}, \quad \text { and } q_{3}^{\lambda, \alpha \beta \gamma}=a_{13}^{\lambda, \alpha \beta \gamma} / 2 a_{20}^{\lambda \lambda} .
\end{aligned}
$$

The first two terms on the rhs of Eq. (A3) constitute the static ZPVA correction for $\beta$, i.e., $\Delta \beta_{\alpha \beta \gamma}^{\mathrm{ZPVA}}(0 ; 0,0)$. By comparing with the BK perturbation treatment at infinite frequency, one can verify that the remaining terms on the rhs of Eq. (A3) are equal to $[\mu \alpha]_{\omega \rightarrow \infty}^{\mathrm{II}}$. This completes the derivation of Eq. (15).

\footnotetext{
${ }^{1}$ Theoretical and Computational Modeling of NLO and Electronic Materials, edited by S. P. Karna and A. T. Yeates, ACS Symposium Series Vol. 628 (ACS, Washington, DC, 1996).

${ }^{2}$ Chem. Rev., Thematic issue on Optical Nonlinearities in Chemistry, edited by J. Michl (1994).

${ }^{3}$ Molecular Nonlinear Optics, Materials, Physics and Devices, edited by J. Zyss (Academic, New York, 1993).

${ }^{4}$ B. Kirtman and M. Hasan, J. Chem. Phys. 96, 470 (1992).

${ }^{5}$ E. A. Perpète, B. Champagne, and B. Kirtman, J. Chem. Phys. 107, 2463 (1997).

${ }^{6}$ D. M. Bishop, B. Champagne, and B. Kirtman (in preparation).

${ }^{7}$ B. Champagne and B. Kirtman (in preparation).

${ }^{8}$ D. M. Bishop and B. Kirtman, J. Chem. Phys. 95, 2646 (1991).

${ }^{9}$ D. M. Bishop and B. Kirtman, J. Chem. Phys. 97, 5255 (1992).

${ }^{10}$ B. J. Orr and J. F. Ward, Mol. Phys. 20, 513 (1971).

${ }^{11}$ D. M. Bishop, B. Kirtman, and B. Champagne, J. Chem. Phys. 107, 5780 (1997).

${ }^{12}$ B. Champagne, E. A. Perpète, J-M. André, and B. Kirtman, Synth. Met. 85, 1047 (1997).

${ }^{13}$ M. del Zoppo, C. Castiglioni, G. Zerbi, M. Rui, and M. Gussoni, Synth. Met. 51, 135 (1992).

${ }^{14}$ D. M. Bishop, J. Pipin, and B. Kirtman, J. Chem. Phys. 102, 6778 (1995).

${ }^{15}$ D. M. Bishop, B. Kirtman, H. A. Kurtz, and J. E. Rice, J. Chem. Phys. 98, 8024 (1993).

${ }^{16}$ J. M. Luis, J. Martí, M. Duran, J. L. Andrés, and B. Kirtman, J. Chem. Phys. 108, 4123 (1998).

${ }^{17}$ J. M. Luis, M. Duran, and J. L. Andrés, J. Chem. Phys. 107, 1501 (1997).
}

${ }^{18}$ J. M. Luis, J. Martí, M. Duran, and J. L. Andrés, J. Chem. Phys. 102, 7573 (1995).

${ }^{19}$ B. Champagne, Chem. Phys. Lett. 287, 185 (1998).

${ }^{20}$ B. Kirtman, B. Champagne, and D. M. Bishop (in preparation).

${ }^{21}$ E. A. Perpète, J.-M. André, and B. Champagne (submitted).

${ }^{22}$ J. M. Luis, J. Martí, M. Duran, and J. L. Andrés, Chem. Phys. 217, 29 (1997).

${ }^{23}$ J. L. Andrés, J. Bertrán, M. Duran, and J. Martí, J. Phys. Chem. 98, 2803 (1994).

${ }^{24}$ J. Martí, J. L. Andrés, J. Bertrán, and M. Duran, Mol. Phys. 80, 625 (1993).

${ }^{25}$ J. Martí, J. L. Andrés, J. Bertrán, and M. Duran, Int. J. Quantum Chem. 52, 9 (1994)

${ }^{26}$ B. Champagne, H. Vanderheoven, E. A. Perpète, and J.-M. André, Chem. Phys. Lett. 248, 301 (1996).

${ }^{27}$ M. J. Cohen, A. Willetts, R. D. Amos, and N. C. Handy, J. Chem. Phys. 100, 4467 (1994).

${ }^{28}$ M. G. Papadopolous, A. Willetts, N. C. Handy, and A. D. Buckingham, Mol. Phys. 85, 1193 (1995).

${ }^{29}$ M. G. Papadopolous, A. Willetts, N. C. Handy, and A. E. Underhill, Mol. Phys. 88, 1063 (1996).

${ }^{30}$ D. M. Bishop, J. M. Luis, and B. Kirtman, J. Chem. Phys. 108, 10013 (1998), following paper.

${ }^{31}$ J. Martí and D. M. Bishop, J. Chem. Phys. 99, 3860 (1993).

${ }^{32}$ D. M. Bishop, Adv. Chem. Phys (to be published).

${ }^{33}$ D. M. Bishop, M. Hasan, and B. Kirtman, J. Chem. Phys. 103, 4157 (1995).

${ }^{34}$ In BK the terms are divided into various types depending on the electrical property derivatives that are involved. See particularly, Eqs. (5)-(9) of Ref. 8.

${ }^{35}$ D. M. Bishop and E. K. Dalskov, J. Chem. Phys. 104, 1004 (1996).

${ }^{36}$ J. M. Luis, M. Duran, J. L. Andres, B. Champagne, and B. Kirtman (in preparation).

${ }^{37}$ H. Sekino and R. J. Bartlett, J. Chem. Phys. 94, 3665 (1991).

${ }^{38}$ D. M. Bishop and S. P. A. Sauer, J. Chem. Phys. 107, 8502 (1997)

${ }^{39}$ D. M. Bishop and J. Pipin, Phys. Rev. A 36, 2171 (1987).

${ }^{40}$ D. M. Bishop, J. Pipin, and S. M. Cybulski, Phys. Rev. A 43, 4845 (1995).

${ }^{41}$ Y. Luo, H. Agren, O. Vahtras, P. Jørgensen, V. Spirko, and H. Hettema, J. Chem. Phys. 98, 7159 (1993).

${ }^{42}$ V. Spirko, Y. Luo, H. Agren, and P. Jørgensen, J. Chem. Phys. 99, 9815 (1993). 